\title{
ESCALAS DE EXPLORAÇÃO VOCACIONAL (EEV) PARA UNIVERSITÁRIOS
}

\author{
Marco Antônio Pereira Teixeira* \\ Marúcia Patta Bardagi \\ Claudio Simon Hutz
}

\begin{abstract}
RESUMO. O comportamento exploratório tem sido descrito na literatura como um aspecto fundamental do desenvolvimento de uma carreira ao longo do ciclo vital. O objetivo deste estudo foi criar um instrumento para avaliar as duas dimensões principais do comportamento exploratório vocacional (exploração de si mesmo e exploração do ambiente) em universitários. O instrumento foi aplicado a 384 universitários com idade média de 21,7 anos, dos quais $65 \%$ eram mulheres. Análises dos componentes principais permitiram identificar as duas dimensões postuladas, demonstrando validade do construto. Os índices de consistência interna observados $(0,85$ e 0,89$)$ também foram satisfatórios. Não houve diferenças de sexo para exploração de si e do ambiente. Estudantes em fim de curso apresentaram níveis mais elevados de exploração do ambiente do que os de início de curso. Embora ainda sejam necessários estudos complementares, os resultados desta pesquisa indicam que este instrumento construído apresenta evidências iniciais de validade e fidedignidade.
\end{abstract}

Palavras-chave: exploração vocacional, estudantes universitários, carreira.

\section{VOCATIONAL EXPLORATION SCALES (VES) FOR UNIVERSITY STUDENTS}

\begin{abstract}
In the international literature exploratory behavior has been described as a fundamental aspect of lifelong career development. The aim of this study was to develop a scale to measure the two main dimensions of vocational exploration (self exploration and environmental exploration) for university students. The scale was applied to 384 university students (mean age 21.7 years), of which $65 \%$ were females. Two dimensions were identified by analysis of principal components and demonstrated the feasibility of the construct. Internal consistency scores ( 0.85 and 0.89$)$ were found to be satisfactory. No significant gender differences in the context of self or of environmental exploration were found. Final year students showed higher environmental exploration than those from the first years. Although complementary studies are still required, results suggest that the scale is valid and reliable.
\end{abstract}

Key words: Vocational exploration, university students, career.

\section{ESCALAS DE EXPLORACIÓN VOCACIONAL (EEV) PARA UNIVERSITARIOS}

RESUMEN. El comportamiento exploratorio ha sido descrito en la literatura como un aspecto fundamental del desarrollo de una carrera a lo largo del ciclo vital. El objetivo de este estudio fue el de crear un instrumento para evaluar las dos dimensiones principales del comportamiento exploratorio vocacional (exploración de sí mismo y exploración del ambiente) en universitarios. El instrumento fue aplicado a 384 universitarios con edad media de 21,7 años, de los cuales el $65 \%$ eran mujeres. Análisis de los componentes principales permitieron identificar las dos dimensiones postuladas, demostrando validad del constructo. Los índices de consistencia interna observados $(0,85$ y 0,89$)$ también fueron satisfactorios. No hubo diferencias de género para exploración de sí y del ambiente. Estudiantes concluyentes de curso presentaron niveles más elevados de exploración del ambiente si comparados a los que estaban a inicio de curso. Aunque todavía sean necesarios estudios complementares, los resultados de esta investigación indican que este instrumento construido presenta evidencias iniciales de validad y fehacientes.

Palabras-clave: exploración vocacional, estudiantes universitarios, carrera.

* Doutor em Psicologia. Professor da Universidade Federal do Rio Grande do Sul.

\# Doutor em Psicologia. Professor da Universidade Luterana do Brasil, campus Santa Maria-RS. 
O comportamento exploratório é fundamental ao desenvolvimento humano, pois através dele conhecemos o mundo e a nós mesmos de uma maneira ativa. No âmbito do desenvolvimento vocacional, mais especificamente, este tipo de comportamento cumpre um papel importante, pois possibilita a reunião de informações essenciais à formação do autoconceito (geral e vocacional) e facilita a organização da experiência, o que por sua vez está associado a maior maturidade de carreira (Flum \& Blustein, 2000).

$\mathrm{O}$ conceito de comportamento exploratório vocacional, inicialmente sistematizado por Jordaan (1963), tem sua origem no campo da psicologia experimental, e designa um comportamento que possibilita $o$ acesso a informações e facilita $o$ aprendizado. É, para o autor, essencialmente um comportamento de solução de problemas, proposital e voluntário, cujo objetivo é reunir determinadas informações sobre o próprio sujeito ou sobre o meio e verificar ou encontrar subsídios para hipóteses que auxiliem o indivíduo a escolher, preparar, assumir, ajustar-se ou progredir em uma ocupação ou carreira. Essa busca de informações envolve experimentação, investigação, tentativa e teste de hipóteses, entre outros comportamentos.

A exploração vocacional foi inicialmente descrita e estudada como um estágio de desenvolvimento. Super (1963) incorporou o conceito de exploração a sua abordagem evolutiva do desenvolvimento vocacional, nomeando a segunda etapa do desenvolvimento de carreira como etapa exploratória. $\mathrm{O}$ autor defendia que a exploração é um comportamento que acompanha o desenvolvimento vocacional, mas é mais característico da adolescência, tendo em vista a natureza das tarefas evolutivas a que o indivíduo está sujeito nesta fase. A atividade exploratória teria por objetivo desenvolver preferências antes da efetivação de uma escolha profissional e da entrada no mundo do trabalho, sendo voltada tanto para o interior (self exploration) quanto para o exterior (environmental exploration) do indivíduo (Super, 1963). Ao analisar as duas dimensões do comportamento exploratório, observa-se uma correlação positiva entre elas (Bartley \& Robitschek, 2000; Sparta, 2003; Werbel, 2000), uma vez que a exploração do ambiente ocupacional leva à necessidade de análise dos próprios valores e interesses e, de outro lado, a auto-análise das habilidades, necessidades e valores leva a uma busca por experiências e oportunidades que venham ao encontro destas características.

Flum e Blustein (2000) enfatizam que a exploração deve ser vista, modernamente, não como um estágio, mas como um processo com função adaptativa para o indivíduo não só no âmbito vocacional. Este processo engloba comportamentos específicos de busca de informações sobre si mesmo e o mundo circundante e um componente atitudinal, referente à motivação para se engajar em atividades exploratórias e mantê-las. Ainda, os autores incluem na definição de exploração não apenas os comportamentos sistemáticos e intencionais iniciados pelo sujeito, mas também aqueles decorrentes de eventos inesperados ou não planejados, mas que produzem informação que contribui para o desenvolvimento de carreira.

A exploração é, destarte, um processo que sustenta a formação do autoconceito, configurando um comportamento que é constante ao longo da vida, mas se intensifica nos momentos que antecedem e seguem períodos de mudança pessoal ou de carreira (Blustein, 1997; Jordaan, 1963; Super, 1963). Pode-se pensar, assim, que os períodos imediatamente anteriores à entrada na universidade e próximos ao término do curso sejam propícios a uma maior atividade exploratória (Nevill \& Super, 1988). De forma geral, o período da graduação como um todo, por ser um momento de implementação de escolhas e realização de novas miniescolhas (estágios, ênfases, áreas de interesse), demanda do aluno um certo nível de comportamento exploratório que possa subsidiar as decisões tomadas ao longo do curso e permita a formulação de projetos profissionais. No entanto, ao contrário do que ocorre no âmbito internacional, apesar da importância do comportamento exploratório para o desenvolvimento vocacional, no Brasil são raros os estudos que investigam as características deste comportamento em adolescentes e adultos.

Uma revisão não extensiva de pesquisas empíricas relativas à exploração vocacional corrobora a importância dada a este construto pelos teóricos do desenvolvimento vocacional. A exploração tem sido sistematicamente correlacionada à maior decisão de carreira (Blustein, Pauling, DeMania \& Faye, 1994; Magalhães, 1995; Sparta, 2003; Teixeira, 2002), autoeficácia vocacional (Bartley \& Robitschek, 2000; Frischenbruder, 1999; Ryan, Solberg \& Brown, 1996), desenvolvimento de expectativas realistas, comportamento de busca de oportunidades (Phillips \& Blustein, 1994; Werbel, 2000), estabelecimento na carreira (Jepsen \& Dickson, 2003) e traços de personalidade, como extroversão, abertura à experiência e conscienciosidade (Reed, Bruch \& Haase, 2004).

No que se refere às possíveis diferenças de gênero na exploração, a literatura tem apresentado resultados 
inconsistentes. Enquanto alguns estudos sugerem a maior exploração feminina (Frischenbruder, 1999; Sparta, 2003; Vignoli, Croity-Belz, Chapeland, Fillipis \& Garcia, 2005), outros indicam o contrário (Blustein \& cols., 1994) ou não apontam diferenças (Ketterson \& Blustein, 1997). Os estudos que observaram maior exploração feminina sugerem que as mulheres tendem a se engajar de forma mais sistemática e deliberada em atividades exploratórias; já os que encontraram escores mais altos de exploração entre os homens argumentam que estes percebem mais oportunidades de trabalho e menos barreiras no mundo profissional, o que estimularia a exploração. Ao considerar a idade, também os resultados não são conclusivos, mas há indícios de que a exploração aumenta à medida que o tempo passa (Frischenbruder, 1999; Ketterson \& Blustein, 1997; Sparta, 2003). Isso pode ser devido ao fato de que os próprios contextos de vida vão se complexificando e exigem maior exposição e busca de informações por parte do indivíduo.

$\mathrm{Na}$ literatura brasileira, são poucos os estudos que contemplam o fenômeno da exploração. Magalhães (1995), em uma pesquisa qualitativa com adolescentes indecisos, observou que os participantes mais indecisos apresentavam dificuldades tanto para explorar características pessoais quanto para elaborar seu autoconceito e traduzi-lo em termos de atributos pessoais vocacionalmente relevantes, o que os levava a tomadas de decisões mais impulsivas e baseadas na estratégia de tentativa e erro. Sparta (2003), em um estudo quantitativo com alunos da terceira série do ensino médio, também encontrou fortes correlações negativas entre a indecisão vocacional e as diferentes dimensões de exploração avaliadas. Em outro estudo com adolescentes, Frischenbruder (1999) observou que a maioria dos participantes explorava o mundo profissional (e pessoal) de forma pouco sistemática e não intencional, sugerindo que a busca de informações vocacionais na adolescência se dá em muitos casos de forma casual, sem seguir um planejamento estruturado. Estes são adolescentes que, ao entrarem no ensino superior, possivelmente correm maiores riscos de se decepcionar e construir uma trajetória de desengajamento do curso e da instituição. Além disso, persistindo um padrão empobrecido de comportamento exploratório na universidade, é provável que a construção da carreira ao longo do ensino superior e a posterior transição para o mercado de trabalho se dêem também de modo fortuito, o que pode vir a frustar as expectativas profissionais dos estudantes (Teixeira, 2002).

Estudos realizados especificamente com universitários mostram que ser capaz de tomar decisões eficientes está relacionado à busca sistemática de informações sobre as próprias habilidades e interesses e sobre oportunidades educacionais e ocupacionais (Werbel, 2000). No contexto brasileiro, Teixeira (2002) identificou relações entre maior comportamento exploratório e otimismo para a busca de emprego entre formandos universitários, além de uma correlação entre exploração e nível de decisão de carreira.

Destarte, avaliar o modo como os universitários exploram suas possibilidades vocacionais, tanto em seus aspectos internos (exploração de si) quanto nos externos (exploração do ambiente), pode ser um recurso importante para se compreender melhor a trajetória dos estudantes ao longo de sua formação e também o percurso profissional após a saída da universidade. Nesse sentido, vale destacar que cada vez mais a literatura brasileira vem se interessando pelo tema da vivência universitária, focalizando aspectos como a adaptação à universidade, a importância das atividades extracurriculares e os processos de permanência e evasão no ensino superior (por exemplo, Joly, Santos \& Sisto, 2005; Mercuri \& Polydoro, 2004). Embora alguns instrumentos venham sendo construídos ou adaptados para avaliar aspectos relacionados à vida universitária no contexto brasileiro (Santos, Noronha, Amaro \& Villar, 2005; Vendramini e cols., 2002), não foram localizados instrumentos nacionais que avaliem mais especificamente o comportamento exploratório vocacional entre universitários.

Sendo assim, o objetivo desta pesquisa foi construir um instrumento, voltado à população universitária, para avaliar as duas grandes dimensões do comportamento exploratório vocacional indicadas na literatura: a exploração de si (que resulta em maior autoconhecimento) e a exploração do ambiente (que resulta em maior conhecimento das alternativas educacionais e profissionais do mundo do trabalho). Além da elaboração de itens para compor este instrumento, pretendeu-se obter evidências de validade fatorial e de consistência interna das escalas.

\section{MÉTODO}

\section{Participantes}

Participaram do estudo 384 universitários de uma universidade federal do RS, com média de idade de 21,7 anos (desvio-padrão de 5,48). As mulheres compuseram $65,1 \%$ da amostra. Os cursos envolvidos foram: Direito $(45,8 \%$ da amostra), Psicologia (44,0\%), Química (5,5\%), Economia $(3,9 \%)$ e outros 
$(0,8 \%)$. Do total, 22,4\% ( $n=86)$ cursavam o primeiro ano do curso, 70,3\% ( $n=270)$ estavam em meio de curso e 7,3\% $(n=28)$ cursavam o último ano.

\section{Instrumento}

Os itens do instrumento foram criados com base nas definições de exploração vocacional de si e exploração vocacional do ambiente, conforme indicado em Stumpf, Colarelli e Hartman (1983) e Flum e Blustein (2000). A exploração de si envolve todo comportamento que produz reflexão ou maior autoconhecimento no que diz respeito a características de personalidade, interesses, habilidades, valores e influências relacionadas à escolha ocupacional. Já a exploração do ambiente engloba comportamentos que visam principalmente a obter informações sobre o mundo ocupacional e do trabalho. Alguns itens foram inspirados no Career Exploration Survey (Stumpf \& cols., 1983), e outros foram elaborados com base na literatura da área de exploração vocacional e da prática em orientação profissional com universitários. Dessa forma, inicialmente, foram criados 26 itens (13 para avaliar exploração do ambiente e 13 para avaliar exploração de si). Os itens da versão final do instrumento encontram-se na Tabela 1. Alguns itens foram eliminados em função das análises realizadas. $\mathrm{Na}$ versão final, os itens de 1 a 13 correspondem à exploração do ambiente e os de 14 a 24 à exploração de si (de acordo com a intenção original das escalas). A chave de respostas utilizada foi uma escala Likert de cinco pontos, com as seguintes opções: 1 - Raramente ou nunca; 2 - Poucas vezes; 3 - Com alguma freqüência; 4 - Freqüentemente; 5 - Muito freqüientemente ou sempre. A pertinência dos itens a cada uma das escalas foi avaliada por dois juizes (psicólogos com experiência no campo da orientação profissional), que julgaram os itens adequados para medir os construtos de exploração de si e do ambiente.

\section{Procedimentos}

A aplicação do instrumento foi feita coletivamente em salas de aula, após esclarecimento sobre a natureza da pesquisa e a obtenção de termos de consentimento dos participantes. A participação foi voluntária, sendo garantidos o sigilo e a confidencialidade dos dados. Este estudo configura-se como um estudo paralelo a um estudo de tese sobre desenvolvimento vocacional de universitários e recebeu aprovação do comitê de etica de uma instituição de ensino superior para sua realização.

\section{RESULTADOS}

\section{Análise da estrutura componencial do instrumento: validade de construto}

A validade de construto de um instrumento referese a quanto esse instrumento é coerente com o conceito ou com a teoria que o embasa. Um procedimento utilizado para verificar a validade de construto de um instrumento é a análise fatorial, que visa a identificar traços latentes que expliquem a variabilidade observada nos itens (Anastasi \& Urbina, 2000; Pasquali, 2003). Se os resultados da análise estiverem de acordo com as expectativas teóricas, então temos alguma evidência da validade de construto do instrumento. Para o instrumento objeto desta pesquisa, esperávamos identificar dois fatores relevantes, correspondentes à exploração de si e do ambiente.

Os 26 itens foram inicialmente submetidos a uma análise de componentes principais. $\mathrm{O}$ índice KayserMeyer-Olkin (KMO) de adequação da amostra foi de 0,89, e o teste de esfericidade de Bartlett mostrou-se significativo $(p<0,001)$, indicando a adequação dos dados para esse tipo de análise. Seis componentes apresentaram autovalores maiores do que 1, explicando $60,7 \%$ da variância total. Contudo, um exame do gráfico de scree sugeriu que apenas os dois primeiros componentes, que explicavam juntos $41,4 \%$ da variância, seriam os mais relevantes. De fato, extraindo-se os seis componentes e procedendo-se a uma rotação oblíqua deles, observou-se que somente os dois primeiros componentes apresentaram pelo menos 6 itens com cargas elevadas (superiores a 0,40). Nos demais componentes, o número máximo de itens com cargas maiores do que 0,40 foi três. Considerando-se estes primeiros resultados, e também a expectativa teórica de que existissem duas dimensões principais subjacentes ao conjunto dos itens, a análise foi refeita extraindo-se apenas dois componentes.

Nesta segunda análise, as comunalidades dos itens situaram-se entre 0,17 e 0,63. Como da primeira vez, optou-se por uma rotação oblíqua, já que as dimensões de exploração vocacional teoricamente devem estar correlacionadas entre si. Os resultados desta análise indicaram o predomínio dos itens relacionados à exploração do ambiente no primeiro componente (que explicou $30,1 \%$ da variância) e a exploração de si no segundo (explicando 11,3\%). No entanto, dois itens apresentaram cargas componenciais inferiores a 0,30 em ambos os componentes e foram eliminados. Ainda, outro item, que havia sido criado para avaliar exploração de si, 
apresentou carga mais elevada (superior a 0,40) no primeiro componente, juntamente com os outros itens elaborados para medir exploração do ambiente. Os demais itens se carregaram conforme esperado, isto é, com cargas elevadas (maiores do 0,40) no componente esperado e baixas (inferiores a 0,35 ) no outro.

Outra análise foi então realizada excluindo-se os dois itens que haviam apresentado cargas baixas (novamente, extraindo-se dois componentes e utilizando-se rotação oblíqua). O índice $\mathrm{KMO}(0,89)$ e o teste de esfericidade de novo indicaram a pertinência da análise. Desta vez, as comunalidades dos itens situaram-se entre 0,19 e 0,64 . O primeiro componente explicou $31,4 \%$ da variabilidade dos dados e o segundo, $12,1 \%$, totalizando $43,5 \%$ de variância explicada. A Tabela 1 exibe as cargas componenciais por item observadas nesta análise.

Tabela 1. Estrutura Componencial dos Itens das Escalas de Exploração Vocacional.

\begin{tabular}{|c|c|c|}
\hline \multirow[t]{2}{*}{ Itens } & \multicolumn{2}{|c|}{ Componentes } \\
\hline & I & II \\
\hline $\begin{array}{l}\text { 1. Quando ouço falar sobre uma nova atividade que me chama a atenção eu procuro mais informações } \\
\text { sobre ela. }\end{array}$ & 0,55 & \\
\hline $\begin{array}{l}\text { 2. Costumo ler livros (ou revistas), assistir a programas de TV (ou vídeos) ou procurar páginas na } \\
\text { Internet que trazem informações sobre minha profissão ou outras de meu interesse. }\end{array}$ & 0,61 & \\
\hline 3. Eu tenho visitado locais de trabalho para conhecer de perto o dia-a-dia dos profissionais. & 0,55 & \\
\hline $\begin{array}{l}\text { 4. Eu converso com meus pais, professores ou amigos para conhecer mais sobre as possibilidades } \\
\text { profissionais. }\end{array}$ & 0,60 & \\
\hline $\begin{array}{l}\text { 5. Eu tenho buscado obter informações sobre o mercado de trabalho e oportunidades de emprego nas } \\
\text { áreas profissionais de minha preferência. }\end{array}$ & 0,80 & \\
\hline 6. Quando procuro informações sobre uma atividade, eu também busco descobrir quais são os seus & 0,66 & \\
\hline
\end{tabular}
possíveis aspectos negativos.

7. Eu tenho procurado conhecer as diversas possibilidades de atuação profissional que existem na $\quad 0,77$ minha profissão (coisas diferentes que um mesmo profissional pode fazer).

8. Eu tenho buscado informações sobre quanto ganham realmente os profissionais que atuam nas áreas 0,54 que me interessam.

9. Eu procuro conhecer as possibilidades de crescimento profissional que existem na minha profissão $\quad 0,70$ ou em outras que me atraem.

10. Eu tenho procurado me informar sobre as principais dificuldades encontradas pelos profissionais da minha área de interesse.

11. Eu tenho tentado conhecer o máximo que eu posso sobre as diversas atividades profissionais 0,66 que existem na atualidade.

12. Eu procuro me atualizar sobre as novas tendências do mercado de trabalho como um todo. $\quad 0,68$

13. Tenho realizado cursos ou assistido a palestras relativas à minha profissão.

14. Eu me coloco em situações que são novas para mim com o objetivo de me conhecer melhor através de experiências diferentes.

15. Eu tenho buscado oportunidades para exercitar as habilidades referentes à minha profissão.

16. Eu tenho parado para pensar sobre que tipos de atividades profissionais realmente me interessam.

17. Costumo pensar sobre quais são minhas principais habilidades e limitações.

18. Tenho avaliado meus interesses e preferências profissionais.

19. Eu tenho pensado na forma como o meu passado se relaciona com a minha escolha profissional.

20. Eu busco refletir sobre as minhas experiências pessoais para aprender mais sobre mim mesmo.

21. Eu tenho me questionado sobre o que eu realmente considero importante em uma profissão.

22. Eu tenho refletido sobre minha história pessoal quando penso sobre o meu futuro profissional.

23. Eu tenho pensado sobre como o meu jeito de ser pode estar relacionado com as minhas preferências profissionais.

24. Eu tenho refletido sobre como as habilidades que eu tenho combinam com as atividades que me interessam.

Nota: são exibidas apenas as cargas de valor superior a 0,30 . 
Como se pode observar, a solução obtida é bastante nítida e coerente com as expectativas teóricas. Originalmente, esperava-se que os itens de 1 a 13 correspondessem à exploração do ambiente, e os de 14 a 24 à exploração de si. De fato, a maioria dos itens de exploração do ambiente carregarou-se no primeiro componente, e os de exploração de si no segundo. A exceção foi o item 15 , que apresentou carga elevada no componente relacionado à exploração do ambiente, quando se esperava que contribuísse mais para a exploração de si. Contudo, uma análise mais detida do conteúdo desse item ("Eu tenho buscado oportunidades para exercitar as habilidades referentes à minha profissão") nos mostra que ele se refere a comportamentos que de algum modo possibilitam ao sujeito obter informações sobre o mundo profissional, talvez mais do que um autoconhecimento. Por isso, decidiu-se mantê-lo no instrumento, mas aproveitando-o como indicador de exploração do ambiente.

\section{Composição das escalas, fidedignidade e estatísticas descritivas}

A partir dos resultados da análise de componentes principais foram compostas duas escalas: a de exploração do ambiente (itens 1, 2, 3, 4, 5, 6, 7, 8, 9, 10, 11, 12, 13 e 15) e a de exploração de si (itens 14, 16, 17, 18, 19, 20, $21,22,23,24)$. O cômputo dos escores individuais nas escalas de exploração foi feito somando-se a pontuação bruta dada pelos sujeitos a cada item em cada escala. A fidedignidade das escalas foi avaliada pelo índice de consistência interna alpha de Cronbach, foi 0,89 para exploração do ambiente e 0,85 para exploração de si. As correlações item-restante corrigidas observadas para as escalas variaram entre 0,37 e 0,72 (exploração do ambiente) e 0,42 e 0,68 (exploração de si), indicando que todos os itens possuem uma correlação aceitável com as escalas de que fazem parte. A Tabela 2 apresenta as médias e os desvios-padrão para as variáveis exploração de si e do ambiente para os grupos: amostra total, homens, mulheres, estudantes em início de curso (até o quarto semestre) e estudantes em fim de curso (do quinto semestre em diante).

Tabela 2. Médias (Desvios-Padrão) das Escalas de Exploração Vocacional

\begin{tabular}{lccc}
\hline \multicolumn{1}{c}{ Grupo } & $\boldsymbol{n}$ & $\begin{array}{c}\text { Exploração do } \\
\text { ambiente }\end{array}$ & $\begin{array}{c}\text { Exploração } \\
\text { de si }\end{array}$ \\
\hline Total & 384 & $49,1(9,21)$ & $45,2(7,38)$ \\
Homens & 134 & $49,2(9,89)$ & $45,0(7,66)$ \\
Mulheres & 250 & $49,0(8,84)$ & $45,3(7,25)$ \\
Primeiro ano & 86 & $46,2(8,89)$ & $44,2(6,12)$ \\
Meio de curso & 270 & $49,5(9,14)$ & $45,4(7,89)$ \\
Último ano & 28 & $54,3(9,10)$ & $46,1(5,58)$ \\
\hline
\end{tabular}

Não foram observadas diferenças estatisticamente significativas (através do teste $t$ de Student) entre os sexos, tanto para exploração do ambiente $(t=-0,21$; g.l.=382; $p<0,84)$ quanto para exploração de si $(t=0,50 ;$ g.l. $=382 ; p<0,62)$. Já ao se compararem os estudantes de primeiro ano, meio de curso e último ano através de análise de variância, observou-se uma diferença significativa para exploração do ambiente $[F(2,381)=9,45 ; p<0,001]$, mas não para exploração de si $[F(2,381)=1,15 ; \quad p=0,317]$. Testes a posteriori (Tukey) mostraram que a diferença em exploração do ambiente deu-se no sentido esperado, ou seja, estudantes de último ano apresentaram escores de exploração significativemente mais elevados do que os de meio de curso, que por sua vez obtiveram escores mais altos do que estudantes de primeiro ano.

Complementarmente, foram realizadas análises correlacionais entre as escalas de exploração e destas com a variável idade (utilizando-se o índice $r$ de Pearson). Observou-se uma correlação de 0,55 $(p<0,001)$ entre exploração do ambiente e exploração de si (considerando-se os escores obtidos com a soma dos itens de cada escala). Correlações próximas a zero foram encontradas entre idade e exploração de si $(r=0,09)$ e idade e exploração do ambiente $(r=0,05)$.

\section{CONCLUSÃO}

O presente estudo teve por objetivo construir um instrumento para avaliar as dimensões exploração do ambiente e exploração de si do comportamento exploratório vocacional. Os resultados obtidos com a análise de componentes principais sugeriram a existência de duas dimensões subjacentes ao conjunto de itens, correspondendo às expectativas teóricas. Estes resultados conferem, portanto, validade fatorial ao instrumento elaborado, ao permitirem a identificação das escalas de exploração do ambiente e de si. Isto significa ainda que, embora as dimensões tenham apresentado correlação (Bartley \& Robitschek, 2000; Sparta, 2003; Werbel, 2000), exploração do ambiente e exploração de si são construtos distintos, e devem ser avaliados separadamente, pois podem ter efeitos diferenciados no desenvolvimento vocacional. Quanto à fidedignidade, os indicadores de consistência interna foram satisfatórios, indicando que as escalas são confiáveis para a avaliação dos construtos a que se referem.

O fato de não terem sido observadas diferenças entre homens e mulheres não chega a surpreender, pois esse resultado já foi relatado, anteriormente, na literatura (Ketterson \& Blustein, 1997). Pode-se pensar 
que, no ambiente universitário, a exploração (tanto do ambiente quanto de si) seja fomentada, principalmente, pelo próprio contexto dos cursos (suas disciplinas, estágios, discussões sobre áreas de atuação e mercado de trabalho, etc); uma vez que ambos os sexos estão sob a influência desse contexto, é compreensível que os níveis de comportamento exploratório sejam semelhante. Estudos nacionais têm encontrado maior exploração em amostras femininas, entre população adolescente (Frischenbruder, 1999; Sparta, 2003). São necessárias novas investigações que observem as mudanças no comportamento exploratório feminino e masculino ao longo do tempo, bem como avaliações que permitam mapear as diferenças de sexo na qualidade da atividade exploratória e na satisfação com a informação obtida.

Por sua vez, as diferenças observadas entre estudantes em início e fim de curso na exploração do ambiente foram claras. Essas diferenças podem ser entendidas como resultado da própria trajetória de formação na universidade. De fato, os cursos vão oferecendo aos estudantes, ao longo do tempo, oportunidades de eles irem conhecendo cada vez mais a profissão escolhida, à medida que incluem disciplinas mais específicas e mesmo de caráter prático. Além disso, com o passar do tempo, os alunos também costumam procurar atividades extraclasse que complementem a formação, como grupos de estudos, minicursos, congressos, que de um modo ou de outro propiciem informações sobre o mundo profissional. À medida que o final do curso se aproxima, a exploração também auxilia na busca de oportunidades ocupacionais e na elaboração de projetos para a transição escola - trabalho, demonstrando a relevância do comportamento exploratório em momentos que antecedem mudanças pessoais e de carreira (Blustein, 1997; Jordaan, 1963; Nevill \& Super, 1988; Super, 1963). De certa forma, este resultado confere também validade ao instrumento, pois é coerente com o que se poderia esperar teoricamente.

Por outro lado, é possível pensar que deveriam existir diferenças também no nível de exploração de si entre os estudantes de início e de fim de curso, possivelmente a favor destes últimos, até mesmo em função da correlação observada entre estas duas dimensões; mas esta diferença não se evidenciou. Pode-se pensar que o aumento no nível de exploração de si ao longo do tempo não seja tão pronunciado quanto o aumento na exploração do ambiente, uma vez que estudantes em início de curso possivelmente já tenham apresentado comportamentos exploratórios que visam ao autoconhecimento, em decorrência da mudança pessoal que representa o ingresso na universidade. De qualquer forma, outros estudos são necessários para investigar melhor esse aspecto.

Não houve relação entre idade e exploração, contrariando a tendência da literatura (Frischenbruder, 1999; Ketterson \& Blustein, 1997; Sparta, 2003). No entanto, pode-se pensar que o aumento da exploração acompanha a idade cronológica pela exigência contextual. Neste estudo, há uma relação positiva entre idade/semestre no curso e uma relação positiva entre exploração do ambiente e semestre, mas não uma relação isolada entre exploração e idade. Os alunos mais velhos que estão em etapas posteriores da graduação podem estar sendo incitados à exploração pela demanda social de novas escolhas de carreira e enfrentamento dos desafios ao longo do curso, mais do que por uma questão apenas maturacional.

Em resumo, as escalas de exploração vocacional demonstraram evidências de validade de construto (através do procedimento de análise fatorial) e consistência interna, o que encoraja o seu uso em novos estudos com populações universitárias. A identificação das características do comportamento exploratório em estudantes pode resultar em informações essenciais à elaboração de projetos de intervenção nesta população, tanto no âmbito institucional quanto no aconselhamento de carreira.

Devem-se levar em consideração, não obstante, as limitações deste estudo. Este foi apenas um primeiro estudo exploratório realizado com este instrumento, não sendo possível concluir definitivamente pela sua validade ou eficácia. Além disso, os participantes desta pesquisa eram provenientes de uma única instituição de ensino superior. Neste sentido, novos estudos são necessários a fim de assegurar a pertinência destas escalas para avaliação do comportamento exploratório, especialmente estudos com amostras maiores e mais diversificadas, que contemplem alunos em diferentes contextos de formação. Sugere-se, ainda, a necessidade do desenvolvimento de instrumentos correlatos que possam avaliar o comportamento exploratório em outras etapas do desenvolvimento vocacional e de carreira no Brasil. Ainda, as próprias escalas de exploração vocacional aqui apresentadas necessitam de estudos complementares que ofereçam evidências mais robustas de validade, tais como o uso de análise fatorial confirmatória para verificar a adequação dos dados ao modelo de dois fatores proposto.

\section{REFERÊNCIAS}

Anastasi, A. \& Urbina, S. (2000, $7^{\text {a }}$ ed.). Testagem psicológica. Porto Alegre: Artes Médicas. 
Bartley, D. F. \& Robitschek, C. (2000). Career exploration: A multivariate analysis of predictors. Journal of Vocational Behavior, 56, 63-81.

Blustein, D. L. (1997). A context-rich perspective of career exploration across the life roles. Career Development Quarterly, 45, 260-274.

Blustein, D. L., Pauling, M. L., DeMania, M. E. \& Faye, M. (1994). Relation between exploratory and choice factors and decisional process. Journal of Vocational Behavior, 44, 75-90.

Flum, H. \& Blustein, D. L. (2000). Reinvigorating the study of vocational exploration: A framework for research. Journal of Vocational Behavior, 56, 380-404.

Frischenbruder, S. L. (1999). O desenvolvimento vocacional na adolescência: autoconceito e comportamento exploratório. Dissertação de Mestrado Não-publicada. Programa de PósGraduação em Psicologia, Pontifícia Universidade Católica do Rio Grande do Sul, Porto Alegre.

Jepsen, D. A. \& Dickson, G. L. (2003). Continuity in life-span career development. Career Development Quarterly, 51, 217233.

Joly, M. C. R. A., Santos, A. A. A. dos \& Sisto, F. F. (Orgs.), (2005). Questões do cotidiano universitário. São Paulo: Casa do Psicólogo.

Jordaan, J. P. (1963). Exploratory behavior: The formation of self and occupational concepts. In D. Super, R. Starishevsky, N. Matlin \& J. P. Jordaan (Eds.), Career development: Selfconcept theory: Essays in vocational development (pp. 42-78). New York: College Entrance Examination Board.

Ketterson, T. U. \& Blustein, D. L. (1997). Attachment relationships and the career exploration process. The Career Development Quarterly, 46, 167-178.

Magalhães, M. O. (1995). Perspectiva experiencial da indecisão vocacional em adolescentes. Dissertação de Mestrado Nãopublicada, Programa de Pós-Graduação em Psicologia do Desenvolvimento, Universidade Federal do Rio Grande do Sul. Porto Alegre, RS.

Mercuri, E. \& Polydoro, S. A. J. (2004). O compromisso com o curso no processo de permanência/evasão no Ensino Superior: algumas contribuições. Em E. Mercuri \& S. A. J. Polydoro (Orgs.), Estudante universitário: características e experiências de formação (pp. 219-236). Taubaté, SP: Cabral Editora \& Livraria Universitária.

Nevill, D. D. \& Super, D. E. (1988). Career maturity and commitment to work in university students. Journal of Vocational Behavior, 32, 139-151.

Pasquali, L. (2003). Psicometria: teoria dos testes na Psicologia e na Educação. Petrópolis: Vozes

Phillips, S. D. \& Blustein, D. L. (1994). Readiness for career choices: Planning, exploring and deciding. Career Development Quarterly, 43, 63-73.
Reed, M. B., Bruch, M. A. \& Haase, R. F. (2004). Five-factor model of personality and career exploration. Journal of Career Assessment, 12, 223-238.

Ryan, N. E., Solberg, V. S. \& Brown, S. D. (1996). Family dysfunction, parental attachment, and career search selfefficacy among community college students. Journal of Counseling Psychology, 43, 84-89.

Santos, A. A. A. dos, Noronha, A. P. P., Amaro, C. B. \& Villar, J. (2005). Questionário de Vivência Acadêmica: estudo de consistência interna do instrumento no contexto brasileiro. Em M. C. R. A. Joly., A. A. A dos Santos \& F. F. Sisto (Orgs.), Questões do cotidiano universitário (pp. 159-177). São Paulo: Casa do Psicólogo.

Sparta, M. (2003). A exploração e a indecisão vocacionais em adolescentes no contexto educacional brasileiro. Dissertação de Mestrado Não-publicada, Programa de Pós-Graduação em Psicologia do Desenvolvimento, Universidade Federal do Rio Grande do Sul, Porto Alegre.

Stumpf, S. A., Colarelli, S. M. \& Hartman, K. (1983). Development of the career exploration survey. Journal of Vocational Behavior, 22, 191-226.

Super, D. E. (1963). Vocational development in adolescence and early adulthood: Tasks and behaviors. In D. E. Super, R. Starishevsky, N. Martin \& J. P. Jordaan, Career development: Self concept theory - essays in vocational development (pp.7995). New York: College Entrance Examination Board.

Teixeira, M. A. P. (2002). A experiência de transição entre a universidade e o mercado de trabalho na adultez jovem. Tese de Doutorado Não-publicada, Programa de Pós-graduação em Psicologia do Desenvolvimento, Universidade Federal do Rio Grande do Sul, Porto Alegre.

Vendramini, C. M. M., Santos, A. A. A. dos, Polydoro, S. A. J., Sbardelini, E. T. B., Serpa, M. N. F. \& Natário, E. G. (2004). Construção e validação de uma escala sobre avaliação da vida acadêmica (EAVA). Estudos de Psicologia, 9, 259-268.

Vignoli, E., Croity-Belz, S., Chapeland, V., Fillipis, A. de \& Garcia, M. (2005). Career exploration in adolescents: The role of anxiety, attachment, and parenting style. Journal of Vocational Behavior, 67, 153-168.

Werbel, J. D. (2000). Relationships among career exploration, job search intensity, and job search effectiveness in graduating college students. Journal of Vocational Behavior, 57, 379-394.

Recebido em 17/11/2005 Aceito em 29/06/2006

Endereço para correspondência: Marco Antônio Pereira Teixeira. Rua Ramiro Barcelo, Instituto de Psicologia UFGS, Bairro Santa Cecília, CEP 90035-003, Porto Alegre-RS. E-mail: mapteixeira@ yahoo.com.br 